\title{
The impossibility of international business
}

Book or Report Section

Accepted Version

Casson, M. (2019) The impossibility of international business. In: Van Tulder, R., Verbeke, A. and Jankowska, B. (eds.) International Business in a VUCA world: The Changing Role of States and Firms. Progress in International Business Research (14). Emerald, Bingley, Yorks, pp. 31-40. ISBN 9781838672584 Available at http://centaur.reading.ac.uk/85420/

It is advisable to refer to the publisher's version if you intend to cite from the work. See Guidance on citing.

Publisher: Emerald

All outputs in CentAUR are protected by Intellectual Property Rights law, including copyright law. Copyright and IPR is retained by the creators or other copyright holders. Terms and conditions for use of this material are defined in the End User Agreement. 


\section{www.reading.ac.uk/centaur}

\section{CentAUR}

Central Archive at the University of Reading

Reading's research outputs online 
The Impossibility of International Business [title and running head]

Mark Casson

Department of Economics and Henley Business School, University of Reading, Reading, UK

Abstract

The optimal location of plants by a global firm is analysed for the first time using measures of distance along the spherical surface of Planet Earth. With a uniform distribution of customers an optimal location strategy will normally seek a space-filling configuration of identical areas that are as near circular as possible. The hexagonal space-filling solution for location on an infinite plane cannot be generalised to the surface of a sphere. Different spatial patterns are required for different numbers of plants; these may be based on triangles, squares or pentagons. The chapter reviews the current state of knowledge on the topic, drawing on theories of spherical geometry and regular convex polyhedra, and on applications in physics, chemistry and medicine. Overall, there appears to be no general solution to the problem; only a set of quite different solutions for various special cases. The lack of any general solution to this central problem in international business illustrates the 'impossibility' referred to in the title of this chapter. [162 words]

\section{KEYWORDS: GLOBAL PRODUCTION LOCATION MULTINATIONAL GEOMETRY IMPOSSIBILITY}

\section{Introduction}

This chapter proposes an extension to the Volatility Uncertainty Complexity Ambiguity (VUCA) framework discussed in this book. It introduces a class of questions that have no answers, and problems that have no solutions. They are questions that it is impossible to address using existing paradigms. Such problems are just as serious as those created by the VUCA quartet. There are many problems of this kind. They are not much discussed in the literature because there are no solutions to present. It is important to discuss these problems, however, because they remind us about how much we do not know, and so, by implication, how little we really know.

It could be argued that these insoluble problems have no solutions precisely because of VUCArelated issues. It is undoubtedly true that VUCA factors can create insoluble problems. Jaynes (2003) for example, has suggested that uncertainty and volatility generate problems in statistical inference that have been misrepresented in conventional probability theory, and that have, in fact, never been systematically addressed. Complexity can also create insolubility; it calls for very complicated models which no simple formula can solve; solutions must be derived through algorithmic iteration. As complexity increases, however, 'combinatorial explosion' may occur. With respect to ambiguity, it is difficult to solve a problem when it is unclear exactly what the problem is. In the early days of international business studies, for example, there was considerable confusion between the production plant and the firm, which held back the development of theory. Ambiguity is therefore an important factor in insolubility.

There are, however, some very simple problems that have no known solution purely because the appropriate models and methods do not exist. These problems do not directly involve any of the conventional VUCA factors. They are unambiguous, they do not require elaborate algorithms, and they exist even in a static world of perfect certainty. This chapter examines one such problem in 
depth. In the light of this discussion, it is proposed that 'impossibility' be added to the VUCA quartet, to generate the more pleasing acronym VUCIA.

\section{An impossible problem: the optimal location of plants}

The problem selected for examination concerns a familiar issue which many readers may believe has already been solved. It concerns the optimal location of production plants by an MNE serving a global market. Various special cases have been discussed but the general case has not. In the international business literature discussions of 'location advantage' or 'country-specific advantage' often cite the classic literature on spatial economics and the economics of location (von Thunen, 1826; Weber, 1929; Christaller, 1933; Losch, 1954; Eaton and Curtis, 1975; Samuelson, 1983; Ota and Fujita, 1993). This spatial literature is not just concerned with plant location and market areas, but also with the growth of cities, the spatial variation of agricultural rents, the stability of spatial competition, and so on.

With respect to plant location and market areas the literature typically assumes that transport costs are directly proportional to distance and to volume carried, and that customers are identical and uniformly distributed across the market area. Each production plant incurs a fixed cost and a variable unit cost. Models focusing on a single firm typically assume that firm to be a monopolist. Various assumptions are made about demand; typically the customer has a downward-sloping demand schedule, or merely purchases a single unit for which they have a fixed reservation price. Sometimes the customers pay their own collection and delivery charges and in other cases the producer absorbs them into the price. Sometimes the producer can discriminate between customers according to where they live or how many units they buy, but normally they do not. These assumptions seem quite strong but some of them are fairly reasonable; furthermore, many of the results derived are quite robust, and do not vary greatly depending on which sets of assumptions are applied. This issue is addressed further at the end of the chapter.

The simplest models of this type analyse the location of plants along a line (e.g. a highway or other transport artery.) A typical solution is that plants are strung out along the line at equal distances from each other, with each plant serving the locations which are closest to it. It should be noted, however, that these models often assume a line of infinite length; this avoids having to analyse the plants at the end of the line differently from the plants in the middle, and having to pack the plants into a fixed amount of space; this is a special case of a more serious issue discussed below.

To provide greater realism, the model may be extended to a flat two-dimensional plane. If there is just a single plant then the conventional solution is very simple: the solitary plant serves a circular market area. The argument for circularity is very simple; If the area were not circular but irregular then transport costs could be reduced by switching supplies from the more distant customers on the extremity to areas nearer to the centre but just outside the boundary; as this process continued, the irregular area would become circular.

When there is more than one plant, complications arise. Even if circular market areas are tightly packed spaces will still remain between them. A close look at a wine-rack will demonstrate the problem. These spaces represent customers who receive no supplies, and they imply a loss of revenue to the firm. It is normally efficient to fill these spaces by serving each customer from the nearest plant. This transforms each circular market area into a square one; its surface is 'tiled' with squares.

Square market areas, however, are not as efficient as circular ones, because along each side of a square the corner points are further away from the centre than the mid-point of the side. There are, 
in fact, many ways of tiling a plane with identically-shaped tiles, and so the question arises as to whether there is a tiling solution better than a square. An efficient solution must involve convex tiles, i.e. tiles where any line connecting two points on the tile lies wholly within the tile. Two simple alternatives are a triangle and a hexagon. The most efficient triangle is an equilateral triangle, and the most efficient hexagon is a regular hexagon where all the sides are of equal length. $A$ hexagon is more efficient than a triangle because, compared to a triangle, the corner points along the perimeter are not much further from the centre than are the mid-points of the sides.

Hexagonal tiling of a plane is a well-known solution in location theory (Christaller, 1933), but it suffers from two serious practical defects. First, the surface of the earth is not unbounded; when boundary conditions are applied then hexagons may not work. A constellation of abutting hexagons always has an irregular boundary and so if the actual boundary is regular then fitting hexagons will leave empty spaces.

More fundamentally, the surface of the earth resembles, not a boundless plane, but the surface of a sphere. International business takes place on the spherical surface of Planet Earth. A boundless plane was a reasonable assumption in the early twentieth century when pioneering theorists were analysing the location of cities in the mid-West US, but it is not a reasonable assumption today. Airlines flights use polar routes to cut journey times and telecommunications rely on satellites circling the earth. Furthermore, employing a realistic spherical model of Planet Earth integrates international business into wider debates about climate change, environmental degradation and geo-political conflict. On a more mundane, but very practical level, the rotation of Planet Earth gives night and day, the tilt in its axis of rotation governs seasonality and its speed of motion around the sun defines the length of the year. Differences in latitude govern opportunities for trade in seasonal products whilst differences in longitude govern geographical differences in the timings of the working day. A spherical mode of Planet Earth affords major opportunities for enhancing the practical and policy relevance of IB theory.

\section{Generalisation to three dimensions}

Moving to three dimensions is not only radical in conceptual terms; it also has radical implications for market areas. This is because hexagons do not tile the surface of a sphere. The optimality of hexagons applies only in two dimensions; it does not apply in three. The reason for this is simple but profound. Unlike the infinite plane, the surface of a sphere is closed. As hexagons multiply, the surface of the sphere bends them round so that they begin to meet up with each other from opposite directions, and there is no guarantee that when they meet up they will match. Indeed, they won't.

This opens up the whole question of where production plants will be most efficiently located on the surface of a sphere. No general solution to this problem is known, so far as the author is aware. This is the 'impossibility' referred to at the outset. There are partial solutions, however, some of which are set out below. These partial solutions suggest some plausible conjectures about what the general solution might be like.

The investigation of this three-dimensional problem does not have to begin from scratch. Spherical geometry is a branch of pure mathematics (Briggs and Edmondson, 1906; Van Brummelen, 2013). It is already known to have many applications in the natural sciences, including physics, crystallography, and bio-chemistry (Smith, 1982). There is substantial evidence, for example, that viruses develop their survival power from the spherical optimality of their chemical structure (Chiu, Coulibaly and Metcalf, 2012). Thus international business is, in some respects, just another area of 
application of spherical geometry. There is a significant difference, however, between IB and these natural sciences, in that IB focuses almost exclusively the surface of the sphere whilst natural scientists tend to focus on the location of atoms or sub-atomic particles within the interior of the sphere as well.

Surface and interior are connected, however, by the theory of regular convex polyhedra, which is another branch of pure mathematics (Aleksandrov, 2005; Zaffanella, 2018). The notable eighteenthcentury Swiss mathematician Leonhard Euler made notable contributions to this field (Bradley, 2007). For the purposes of this chapter a regular convex polyhedron may be described as a manysided object where the shape of each side is identical to every other side and all the edges of each side are of the same length. The corners of these regular convex polyhedra all coincide with points lying on the surface of a sphere. This particular type of polyhedron was well known to Greek mathematicians; there are only five of these polyhedra, and they are now known as the Platonic solids (DeHovitz, 2016). For each Platonic solid a set of market areas can be generated by projecting the sides radially from the centre of the sphere onto its surface. Each set will tile the entire surface of the sphere with a set of identically shaped and identically sized market areas.

While all Platonic solids generate markets areas, not all market areas are derived from Platonic solids. With a single plant the entire surface of the earth can be served equally well from any point. Thus one market area is the sphere itself. Note that on a plane the corresponding market area would be potentially infinite, and bounded only by escalating transport costs. On the sphere the market area is finite even if transport costs are zero. There is another feature specific to the sphere which merits comment. A threshold is reached when half the global market is served. If the location of the plant is identified as a pole then the boundary of this area is the equator. With a uniform distribution of customers the equator marks the point where a marginal increase in the distance along the surface from the plant generates the greatest number of additional customers; after this point the number of additional customers declines steadily until a single final customer is served at opposite pole. If transport costs are reasonably high relative to product price, then under certain pricing strategies the equator marks a significant point at which the boundary of the market area may be set.

With two plants, efficiency requires that each plant is located at the opposite pole to the other. The boundary of the market area would be the equator, as described above. The location of these poles is indeterminate, however. This result suggests that under certain conditions a global firm might deliberately choose to locate at the furthest point away from home, rather than nearby. This may explain the apparent paradox that firms headquartered in imperial countries often locate at the furthest point of empire rather than the nearest, e.g. Dutch investment in the East Indies and British investment in Australia (Stopford, 1974).

For three plants there is no known solution. The best that can be achieved is three equal-sized, but not identically-shaped, market areas.

With four market areas the first, and simplest, of the Platonic solids is encountered. The tetrahedron is a symmetric pyramid with a triangular base; each of the three sides has the same size and shape as the base. When projected onto the surface of the encompassing sphere, the triangular pattern is replicated (see Figure 1). The efficiency of this solution is limited by the triangular shape of each market area, for which the distance of the boundary from the centre varies greatly between a vertex (far) and a mid-point of a side (relatively near).

There is no known solution for five plants (although see below). 
The cube has six square sides and when projected each of these sides acquires a curvature dictated by the radius of the sphere. The advantage of the cube is that as the projections are only modestly curved, each side can be readily divided into four areas which are also relatively square (see Figure 2). These four areas meet at a point which is the projection onto the spherical surface of the centre of the relevant side of the underlying cube. Each subdivision has the same basic shape but a different orientation to the central point. This generates $6 \times 4=24$ market areas. Further subdivision is possible, by making four new market areas out of each of the previous 24 market areas, but now the sub-divided areas differ according to which of the first previous sub-divisions they are created from. Furthermore, the areas that lie in the corners of the original six areas are more triangular than other areas that adjoin the centres of these areas.

There is no known solution for seven plants.

An octahedron generates eight equilateral triangular market areas. These eight market areas can also be generated directly on the surface by drawing three great circles that intersect each other at right angles. The first is equivalent to an equator, the second to a meridian through the poles, and the third to another meridian at right angles to the first. The intersections of these three circles generate eight curved equilateral triangles. Each of these triangles can be subdivided into three smaller isosceles triangles, but these have inefficient shapes; the underlying segment of the octahedron has one angle of 120 degrees and two smaller angles of only 30 degrees. Despite its ease of construction, this solution is relatively inefficient compared to the cube.

A dodecahedron is a set of twelve pentagons which generates twelve identical five-sided market areas (see Figure 3). This configuration is more efficient than the six squares generated by the cube. The square sides of the cube have interior angles of 90 degrees, whilst the interior angles of the pentagon are 108 degrees, indicating that its shape is a compromise between a square and a circle (Moulton, Lu and Zaworotko, 2001). The possibilities of economical sub-division are more limited, however, since the pentagon sub-divides naturally into five isosceles triangles with interior angles of 72 degrees, 54 degrees and 54 degrees.

The final Platonic solid is the icosahedron, comprising 20 equilateral triangles, which give twenty triangular market areas when projected onto the surface of a sphere. This configuration is potentially useful when there are many plants each serving relatively small areas. But for reasons already stated the shape of the market areas is not particularly efficient. Further division is not particularly efficient either. It is worth noting, however, that adjacent market areas can be combined. Combining adjacent pairs generates ten six-sided market areas, which can, if desired, be further combined to generate five eight-sided areas. These larger areas provide solutions where none existed previously, but they are not particularly efficient.

\section{Conclusion}

The analysis above has shown that no general solution is known to the optimisation of plant location on the surface of a sphere. This does not mean that there is no solution, but only that such a solution, if it exists, remains to be found. Solutions exist for special cases of the problem. Some numbers of plants support relatively efficient market areas and some do not. Some do not support any configuration of identical market areas at all.

The results relating to Platonic solids can be mathematically proved, but some of the propositions regarding projections onto the sphere remain conjectural in strictly mathematical terms. The preliminary results, though indicative, are important: they suggest that interlocking squares have much to commend them, but they only work with specific numbers of plants. This raises the issue of 
whether certain numbers of plants are more common than others because the geometry of market areas favours them. The results also indicates that triangular market areas, though relatively inefficient, are also very versatile in covering the surface of a sphere; this suggest that triangular market areas may emerge as 'second best' when superior alternatives are not available.

It is possible, of course, to criticise the assumptions on which this analysis is based. The most crucial assumption is that customers are uniformly distributed over the surface of the sphere. This assumption can be questioned at both a macroscopic and microscopic level. At the macroscopic level, it ignores the fact that only a small proportion of the earth's surface is usable by international business. The oceans and rivers function as highways and not as centres of residence or locations of production. Rugged mountain ranges are unsuitable for transport and residence, and their main productive use is mining. Nevertheless the assumption of uniformity provides a useful benchmark against which to assess the impact of natural geography on the location of production. At a microscopic level most consumers agglomerate in urban centres, so that market areas comprise a set of salient points rather than a continuous area.

From the perspective of this chapter, however, these arguments also work in the opposite direction. If the optimal allocation of production is an almost intractable problem even in the simplest possible case, how much complicated is it in practice? The optimal location of production is not only complex; it may also be impossibly complex, and especially so when analysed in terms of Planet Earth.

The main lesson of this chapter, far from being nihilistic, is challenging. There may well be a general solution to the optimal location problem, and if it exists it would be a major intellectual achievement to discover it. But even if it were discovered, it would be challenging to implement in practice. This suggests that simple models will always be required. A general solution in three dimensions will not render one- or two-dimensional models obsolete. One- or two-dimensional models will always be simpler than three-dimensional models, and many points will continue to be made as readily in one or two dimensions as they are in three. Impossibility is always going to be a challenge in a subject as demanding as IB theory. Tackling seemingly impossible problems is part of the fun of the subject, but developing simple formal models in the meantime will ensure that incremental progress continues to be made. 


\section{Acknowledgements}

I am grateful to Peter Buckley, Janet Casson, Jeremy Clegg and Nigar Hashimzade for useful discussions on this topic. 
References

Aleksandrov, A.D. (2005) Convex Polyhedra. Berlin: Springer

Bradley, R. E. (2007) Leonhard Euler: Life, Work and Legacy. Amsterdam: Elsevier

Briggs, William and Edmondson, T.W. (1906) Mensuration and Spherical Geometry, $3^{\text {rd }}$ ed., London: University Tutorial Press

Chiu, E, Coulibaly, F. and Metcalf, P. (2012) Insect virus polyhedra, Current Opinions in Structural Biology, 22(2), 234-240

Christaller, W. (1933) The Central Places of Southern Germany, Englewood Cliffs, NJ: Prentice-Hall

DeHovitz, Deborah C. (2016) The Platonic Solids.

https://www.whitman.edu/Documents/Academics/Mathematics/2016/DeHovitz.pdf, accessed 23/05/2019

Eaton, B C. and Lipsey, R. G. (1975) The principle of minimum differentiation: some new developments in the theory of spatial competition, Review of Economic Studies, 42 (1) 27-49 Jaynes, E.T. (2003) Probability Theory: The Logic of Science, Cambridge: Cambridge University Press Losch, August (1954) (trans. W.H.Woglom) The Economics of Location, New Haven: Yale University Press

Moulton, B., Lu, J., and Zaworotko, M.J. (2001) Periodic tiling of pentagons. Journal of the American Chemical Society, 123 (37), 9224-5

Ota, M. and Fujita, M. (1993) Communication technologies and spatial organization of multi-unit firms in metropolitan areas. Regional Science and Urban Economics, 23 (6), 695-729

Samuelson, P. A. (1983) Thunen at two hundred. Journal of Economic Literature, 21, 1468-1488.

Smith, J. V. (1982) Geometrical and Structural Crystallography. New York: John Wiley

Stopford, J. M. (1974) The origins of British-based manufacturing enterprises, Business History Review, 48(3), 303-335

Van Brummelen, G. (2013) Heavenly Mathematics: The Forgotten Art of Spherical Trigonometry. Princeton, NJ: Princeton University Press

Von Thunen, J.H. (1826) Von Thunen's Isolated State (trans. C.M. Wartenberg). Oxford: Pergamon Press, 1966

Weber, A. (1929) The Theory of the Location of Industries. Chicago: Chicago University Press Zaffanella, E. (2018) On the efficiency of convex polyhedra, Electronic Notes in Theoretical Computer Science,354, 31-44 
Figure 1

Projection of tetrahedron: 'side' view

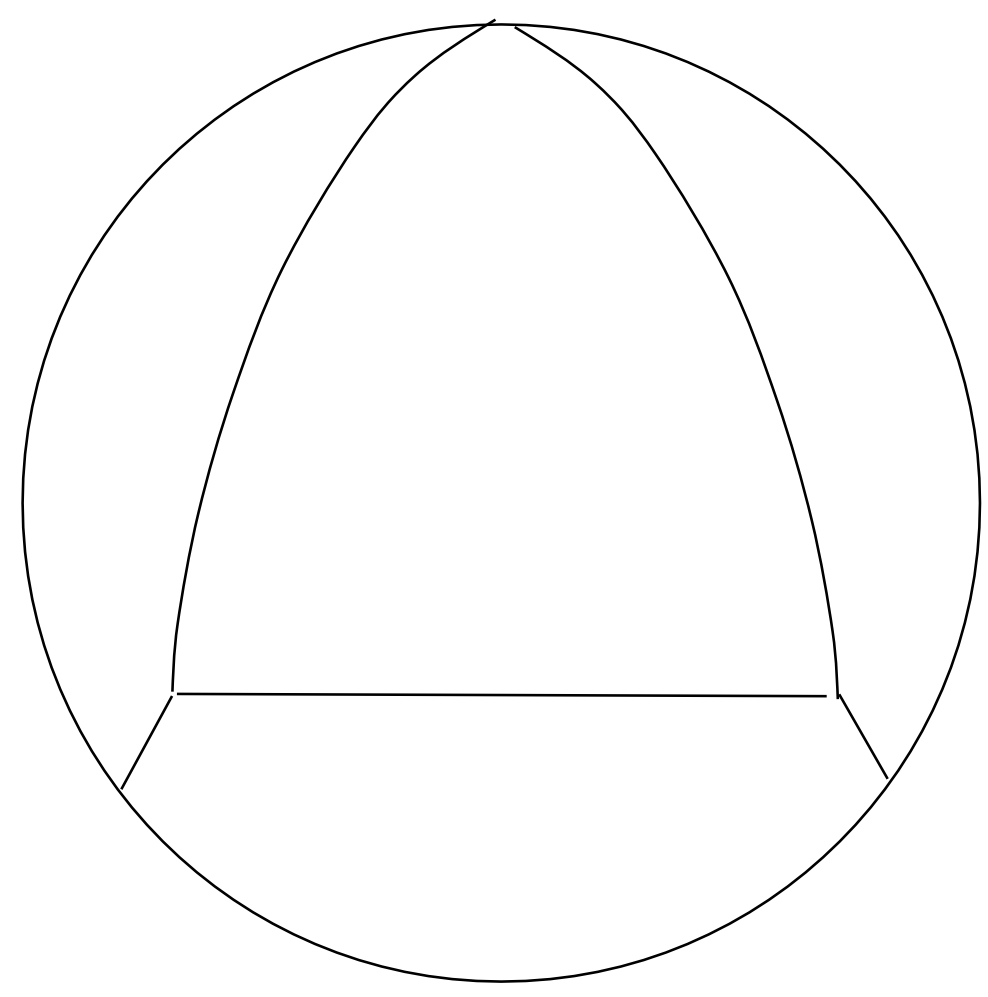

Figure 2

Projection of cube: 'side' view

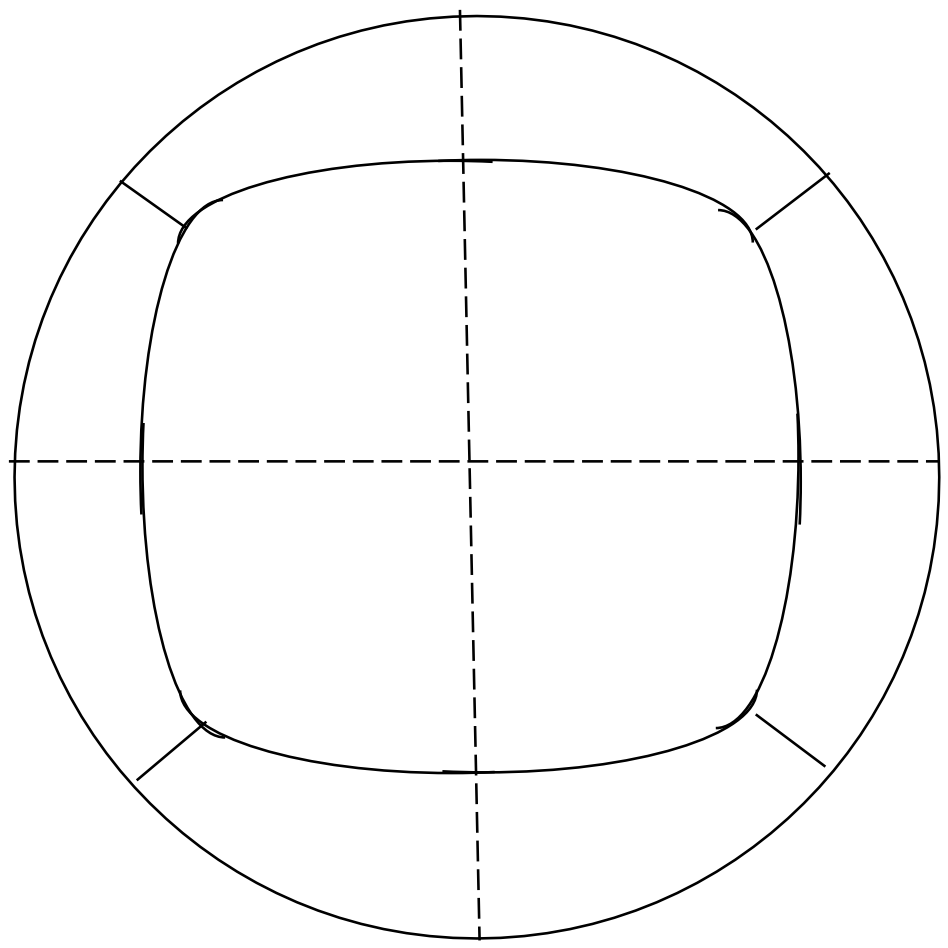


Figure 3

Projection of dodecahedron: 'side' view

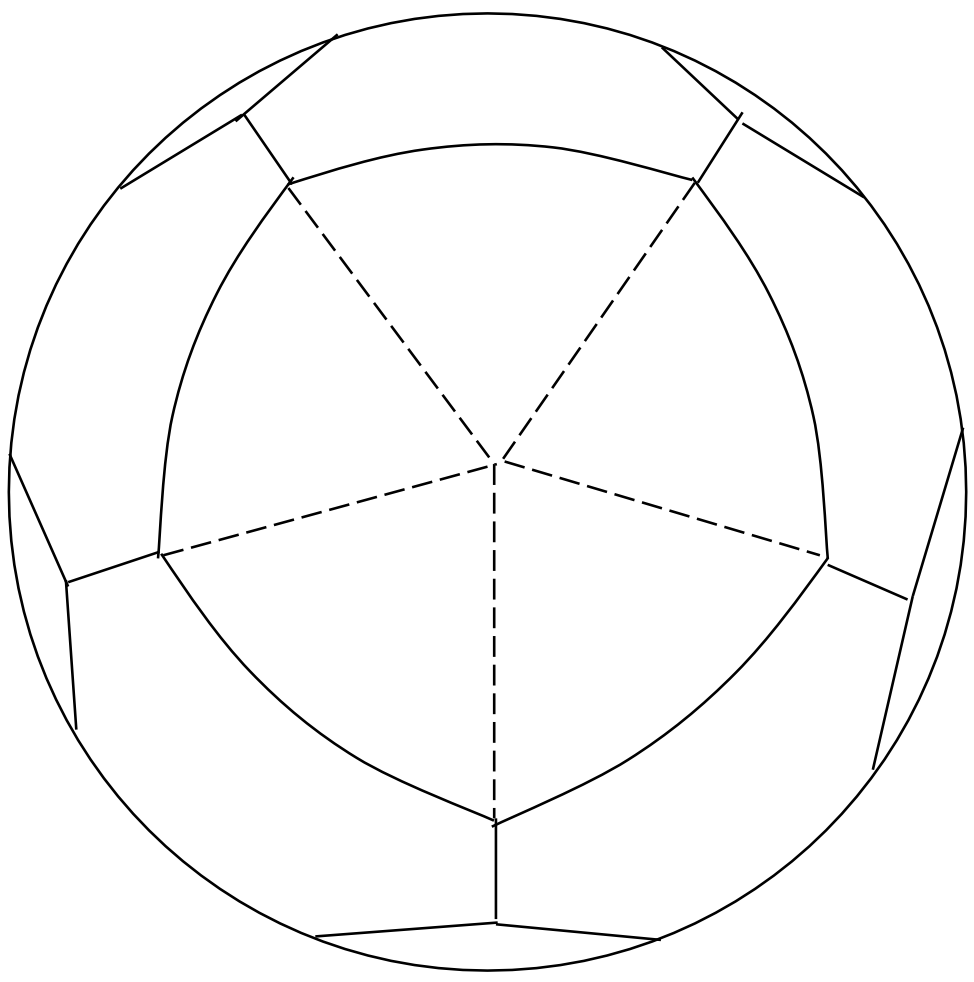

